\title{
(2) OPEN ACCESS \\ Histopathological analysis of in vivo specimens of recurrent aneurysms after coil embolization
}

\author{
Chao Wang, ${ }^{1}$ Mengxing Li, ${ }^{1}$ Huiyuan Chen, ${ }^{2}$ Xinjian Yang $\odot{ }^{1,3}$ Ying Zhang, ${ }^{1}$ \\ Dong Zhang ${ }^{4}$
}

- Additional supplemental material is published online only. To view, please visit the journal online (http://dx.doi. org/10.1136/neurintsurg2021-017872)

${ }^{1}$ Department of Interventional Neuroradiology, Beijing Neurosurgical Institute, Beijing China

${ }^{2}$ Department of Neuropathology, Beijing Neurosurgical Institute, Beijing Tiantan Hospital, Beijing, China

${ }^{3}$ Department of Interventional Neuroradiology, Beijing Tiantan Hospital, Beijing, China

${ }^{4}$ Department of Neurosurgery, Beijing Tiantan Hospital, Beijing, China

Correspondence to

Dr Ying Zhang; yingzhang829@ 163.com

Dr Dong Zhang;

zhangdong0660@aiyun.com

Received 8 June 2021

Accepted 26 July 2021

\section{ABSTRACT}

Background Aneurysm recurrence after coil embolization remains a challenging problem.

Objective To determine the histopathological features of recurrent aneurysm specimens and explore the mechanism of aneurysm recurrence.

Methods Nine aneurysm specimens were collected from eight patients who underwent clipping for aneurysm recurrence within 2 years after embolization. All specimens were sectioned and embedded in resin, stained with hematoxylin-eosin (H\&E), Masson stain, and immunohistochemical staining for smooth muscle actin (SMA) and CD68+ antibodies, and were examined under light microscopy.

Results Five aneurysms were surgically clipped owing to post-embolic subarachnoid hemorrhage, while the other four aneurysms had dangerous recanalization detected on follow-up imaging. Five aneurysms had self-growth and four aneurysms had coil compactions. Gross observation showed that each recurrent aneurysm was wrapped by a thrombus and the aneurysm wall; some coils protruded from the pseudocapsule in some ruptured aneurysms. Microscopically, H\&E staining showed that three types of thrombi (fresh thrombus, granulation tissue, and scar tissue) coexisted in one section. In addition, characteristic unstable and unorganized thrombi with empty spaces were found in the neck cavity. Immunohistochemical staining showed that the SMA stain was discontinued and incomplete, and CD68 + antibody and H\&E staining revealed inflammatory infiltrate in the aneurysm wall.

Conclusion The coexistence of three types of thrombi is the main characteristic of recurrent aneurysms. The formation of stable thrombus may be one of the key points of aneurysm recurrence. Smooth muscle cell damage and infiltration of inflammatory cells in the aneurysm wall probably contribute to the recanalization.

\section{INTRODUCTION}

Unruptured intracranial aneurysms (IAs) are common cerebrovascular conditions. Aneurysm rupture results in subarachnoid hemorrhage (SAH), which is an important subtype of stroke with high mortality and morbidity rates. ${ }^{1}$ Therefore, appropriate intervention is necessary for IAs. ${ }^{2}$ The initial intervention method was microscopic aneurysm clipping. After the development of catheterization techniques with cerebral angiography, endovascular coiling has become another way of treating IAs. Moreover, endovascular therapy improves patient quality of life immediately after treatment and during follow-up, and has a lower mortality rate than surgical clipping. ${ }^{3}{ }^{4}$

Although endovascular therapy has been verified as safe and effective, IA recurrence may occur even after complete coil embolization of the aneurysm. ${ }^{5}$ The recurrence rate is relatively high after coiling alone and after stent-assisted coil embolization. ${ }^{67}$ IAs often recur early after endovascular treatment. Nearly $50 \%$ of IA recurrences occur within 6 months after coiling in humans. ${ }^{8}$ The mechanism of IA recurrence is complex and the specific processes are still unclear. Many hypotheses have been proposed for IA recanalization, including: (1) growth of the aneurysm itself, ${ }^{59}$ (2) coil compaction, ${ }^{510}$ (3) degradation and recanalization of fresh and unstable thrombotic tissue, (4) continuous blood flow through the intraluminal coils and thrombosis complex, ${ }^{11}$ (5) lack of neointima formation across the neck of the aneurysm, ${ }^{12}$ (6) lack of smooth muscle in the IA wall, leading to organized thrombus reduction. ${ }^{1314}$ To solve the problem of IA recurrence, this study investigated the pathology of specimens collected from patients who underwent clipping after unsuccessful coil embolization.

\section{PATIENTS AND METHODS}

Between June 2019 and January 2021, eight patients with nine recurrent saccular aneurysms underwent surgery in our hospital. All patients had received previous embolization treatment before undergoing surgical clipping. Four patients with five recurrent aneurysms experienced SAH after embolization. The other four recurrent aneurysms in four patients were detected on follow-up imaging. In our former study, ${ }^{15}$ recurrent aneurysms were classified into the following five types: I, pure recanalization inside the aneurysm sac; II, pure coil compaction without aneurysm growth; III, new aneurysm neck formed without coil compaction; IV, new aneurysm neck formed with coil compaction; and V, newly formed aneurysm neck and sac. Types I-II can be resolved with endovascular treatment, while types III-V require surgical clipping. This strategy results in a satisfactory cure rate and compactions. In the present study, four recurrent IAs (two type III, one type IV, and one type V) and five ruptured IAs in eight patients were analyzed. Patient and aneurysm information is presented in table 1 .

A standard endovascular technique was used to access and pack the IAs with coils as tightly as possible. Preoperative dual antiplatelet therapy (100 mg aspirin and $75 \mathrm{mg}$ clopidogrel) was used 


\begin{tabular}{|c|c|c|c|c|c|c|c|c|}
\hline $\begin{array}{l}\text { Aneurysm } \\
\text { No }\end{array}$ & First coiling reason & Endovascular treatment times & $\begin{array}{l}\text { Last implant time } \\
\text { (months)* }^{*}\end{array}$ & Clipping reason & Location & $\begin{array}{l}\text { Size/neck } \\
(\mathrm{mm})\end{array}$ & Imaging findings & Typet \\
\hline 1 & Headache & 2 & 2 & aSAH & AComA & $13.3 / 4.5$ & Aneurysm growth & III \\
\hline 2 & Headache & 2 & 2 & aSAH & RMCA & $12.2 / 4.2$ & Aneurysm growth & III \\
\hline 3 & aSAH & 1 & 35 & Recurrence & RMCA & 4.3/2.2 & Coil compaction & IV \\
\hline 4 & Dizziness & 1 & 6 & Recurrence & PComA & $25.0 / 8.4$ & Coil compaction & $\mathrm{V}$ \\
\hline 6 & aSAH & 1 & 7 & Recurrence & AComA & $5.2 / 2.4$ & Aneurysm growth & III \\
\hline 7 & aSAH & 2 & 8 & Recurrence & AComA & $5.3 / 2.2$ & Coil compaction & III \\
\hline 8 & aSAH & 1 & 71 & aSAH & LCA C7 & $6.3 / 2.2$ & Aneurysm growth & III \\
\hline 9 & aSAH & 2 & 111 & aSAH & LCA C7 & $13.4 / 4.5$ & Aneurysm growth and coil compaction & IV \\
\hline
\end{tabular}

*Last implant time means the interval between the last endovascular treatment and clipping.

tThe type of recurrent aneurysm is classified into the following five types: I, pure recanalization inside the aneurysm sac; II, pure coil compaction without aneurysm growth; III, new aneurysm neck formed without coil compaction; IV, new aneurysm neck formed with coil compaction; and V, newly formed aneurysm neck and sac. Types I-II can be resolved with endovascular treatment, whereas types III-V require surgical clipping. AComA, anterior communicating artery; aSAH, aneurysmal subarachnoid hemorrhage; LCA C7, left carotid artery communicating segment; PComA, posterior communicating artery; RCA C7, right carotid artery communicating segment; RMCA, right middle cerebral artery.

for at least 5 days, and a loading dose $(300 \mathrm{mg}$ aspirin and $300 \mathrm{mg}$ clopidogrel once) was used for ruptured aneurysms. Dual antiplatelet therapy was continued for 1 month after treatment, and aspirin was continued for 3 months after treatment in the patients with stenting. For patients accepting elective surgery, antiplatelet medication was discontinued at least 1 week before clipping. For the patients who have an aneurysmal SAH and still take antiplatelet drugs before clipping, the use of aspirin will have an adverse effect on the operation. We will not launch a clipping operation before the patients have withdrawn the antiplatelet drugs for 1 week, unless their condition continues to deteriorate (such as enlargement of intracranial hematoma, aneurysms with daughter sac and a high risk of re-rupture, conscious state continuous deterioration) and they are unable to continue conservative treatment.

The microsurgical clipping was done via the supraorbital approach and the pterional approach. In patients with an IA in the posterior communicating artery, anterior communicating artery, middle cerebral artery, and communication segment of the internal carotid artery, microsurgical clipping was performed through the ipsilateral pterional approach. However, for patients with a less-developed frontal sinus and anterior orientation communicating artery aneurysms, clipping was performed via the ipsilateral supraorbital approach. Successful exclusion of the aneurysm and preservation of the branches was verified using intraoperative indocyanine green video angiography. The detailed surgical procedures are shown in online supplemental figures. Aneurysm specimens were carefully collected during surgery.

Aneurysms were inspected thoroughly after removal. The specimens were fixed in buffered formaldehyde and embedded in resin (methyl methacrylate). Serial sections were made using a diamond wire saw and hand polishing. Specimens were prepared using the standard of creating sections perpendicular to the aneurysm neck. Nine sections were stained with hematoxylineosin (H\&E) and Masson stains. One integral specimen with a larger size was divided into two segments that were resin embedded and paraffin embedded for immunohistochemical and histochemical staining, respectively. All specimens were independently examined by two histopathologists using light microscopy. For each case, the histopathologists recorded signs of incomplete thrombosis (fresh blood, unorganized thrombus, void spaces), inflammatory response (foreign body giant cells, leukocyte, macrophages invasion), and thrombus organization (fibrocellular reaction, collagen formation, neovascularization), as well as the immunohistochemical staining results. Two or three slides were made from each aneurysm. All slides were studied in a similar manner and the most representative slide was selected for analysis. Detailed histological findings are shown in online supplemental table 1, using the histopathology result score ${ }^{16}$ (online supplemental tables 2-6).

\section{RESULTS}

\section{Patient characteristics and imaging findings}

Before the first coiling, six patients with six aneurysms had $\mathrm{SAH}$ due to aneurysm rupture, and the three IAs in the other two patients were detected on imaging. Four patients received the second endovascular treatment for aneurysm rupture or recurrence (two patients were treated with coiling alone, and two were treated with stent-assisted coiling). All eight patients finally underwent surgical clipping because of post-coiling aneurysm recurrence or SAH during follow-up. Four patients with five aneurysms had an aneurysmal SAH and four patients with four aneurysms showed aneurysm recurrence on imaging. Seven patients with seven aneurysms, who did not take the antiplatelet drug, accepted clipping directly. Only one patient with two aneurysms still took aspirin because the stent was placed during last endovascular treatment ( 2 months ago), then accepted conservative treatment until aspirin withdrawal after 1 week, and finally received clipping.

The surgical series included five men and three women with a mean age of $56 \pm 10.3$ years and a mean interval between the last embolization and clipping of $38.2 \pm 44.7$ months (range 2-111 months). The aneurysm location was the anterior communicating artery in three cases, posterior communicating artery in one, internal carotid artery communicating segment in three, and middle cerebral artery in two. The mean aneurysm size was $10.4 \mathrm{~mm}$ (range $4.3-25.0 \mathrm{~mm}$ ) and mean neck width was $3.9 \mathrm{~mm}$ (range $2.2-8.4 \mathrm{~mm}$ ). The recurrence type was selfgrowth for five aneurysms, with a mean maximum size growth of $2.8 \mathrm{~mm}$ (range $2-4 \mathrm{~mm}$ ); four were coil compactions detected on follow-up images (figures 1 and 2). For the aneurysms with coil compactions, this phenomenon was observed through imaging comparisons without detailed quantitative data analysis. Detailed information of the patients and aneurysms is shown in table 1 .

\section{Gross observations}

Six of nine aneurysms had a preserved, intact, yellow-pink, thin wall with coils poking out of the wall and blood clots. The 

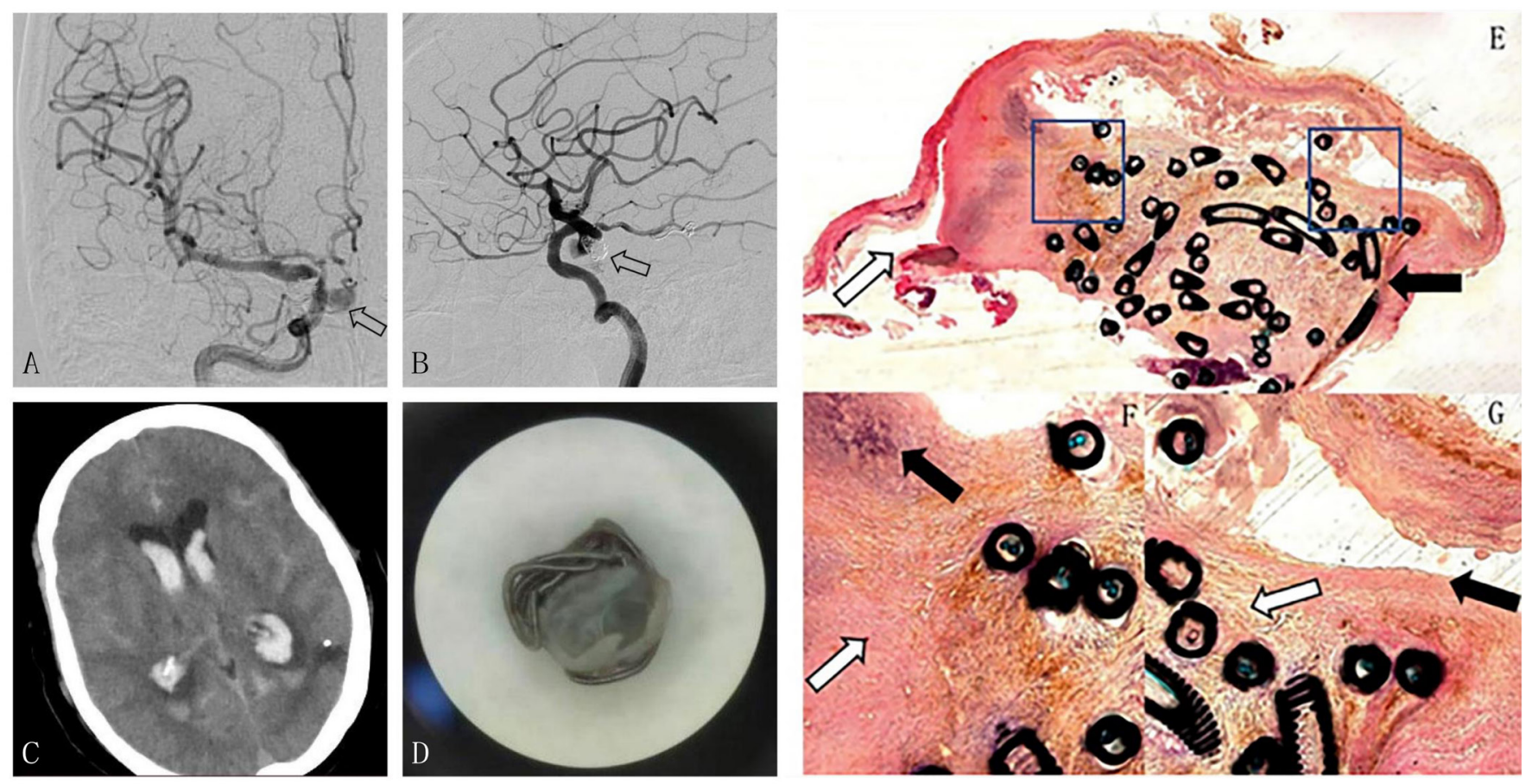

Figure 1 Case 9, patient in their 60s with an aneurysm located at LC7. (A) Cerebral angiography demonstrates complete obliteration of the aneurysm with stent-assisted coiling in secondary treatment (open arrow). (B) Pre-clipping cerebral angiography shows aneurysm recurrence (aneurysm growth and coil compaction of the residual neck and subarachnoid hemorrhage) (open arrow). (C) Pre-clipping head CT shows hemorrhage in the ventricle and fissures. (D) Gross specimen with coils packed by the thrombus and wall. (E-G) Microscopic section (hematoxylin-eosin stain, magnification $\times 4$ and $\times 12.5$ ). (E) Empty space is seen near the aneurysm neck (open arrow), and the coils are attached to the thrombus (arrow). (F) Granulation tissue (open arrow) consisting of the thrombus, fibroblasts, and infiltrating inflammatory cells (arrow). (G) Coils forming a tight junction with scar tissue (mature granulation) (open arrow), which is causing surface angiogenesis (endothelial cell formation) (arrow).

buck of the coils was packaged by the thrombus and aneurysm wall. The neck remnant cavity could be clearly observed, and the neck tissue was thicker than the dome. (figures 1 and 2D). For the other three aneurysms, only incomplete specimens were obtained because the tissue was partly damaged during surgery. Therefore, only the thrombus and coils could be observed.

\section{Histologic findings}

The three types of thrombus found in most aneurysms were fresh, unorganized, and stable. (1) A fresh thrombus was seen in the aneurysm dome where incomplete embolization left empty space within the aneurysm sac. The thrombus consisted of erythrocytes and a small number of macrophages around the fibrillar collagen, with no thrombus attached to the coils. (2) Unorganized and unstable thrombi were located near the empty neck, and were gradually organizing into granulation tissue. (3) Stable thrombi comprised complete scar tissue located in the middle of the sac, close to the aneurysm wall. These were mature thrombi with a layer of endothelial cells formed on the surface (ie, re-endothelialized), and were tightly connected to the coils. Inflammatory cells, including neutrophils, hemosiderin macrophages, and lymphocytes, had infiltrated the aneurysm sac (figure $1 \mathrm{E}-\mathrm{G}$ ). The inflammatory cell infiltration of the wall was also seen on H\&E staining.

In the ruptured aneurysm specimens, H\&E staining showed serum effusion and necrotic cells with stained vacuoles, indicating bleeding in this area. One unfibrosed thrombus was loosely linked with the coils in the center of the section. Except for this thrombus, the other thrombi were composed of transforming scar tissue, with no cells but only necrotic collagen fibers. The inflammatory reaction was similar to the description in the previous paragraph (figure $2 \mathrm{E}-\mathrm{G}$ ). Compared with the unruptured aneurysm specimens, the ruptured specimens had more fresh thrombi and less endothelial lining of the aneurysm wall.

Masson staining resulted in blue-purple coloring surrounding the packing coils in the aneurysm sac, which represented mature and organized thrombi with collagen and mature granulation tissue. The stain was still unsatisfactory after two attempts.

Immunohistochemical staining was performed on one aneurysm using CD68 and smooth muscle actin (SMA) antibodies. Macrophages were distributed throughout the whole vascular wall, especially in the outer layer. SMA staining showed the discontinuity of smooth muscle cells (SMCs) in the middle media layer. The infiltration of inflammatory cells was more severe in areas with less SMA staining, where the inflammatory cells included macrophages and lymphocytes in the aneurysm wall (figure 3A,B).

\section{DISCUSSION}

Coil insertion quickly changes the intra-aneurysm blood flow and decreases the aneurysm mural pressure to reduce the lumen blood flow velocity, contributing to thrombosis embolization and ultimately leading to aneurysm occlusion. ${ }^{17}$ However, aneurysm recurrence after embolization treatment is still very common in clinical practice. We conducted pathological studies on recurrent aneurysms after endovascular therapy and obtained some important findings. The intra-aneurysm thrombosis showed some interesting distribution features after coiling, and granulation tissue was found in the neck of the recurrent aneurysms.

In the histopathological analysis, all thrombus stages were observed in the recurrent aneurysm sac. This finding suggests 

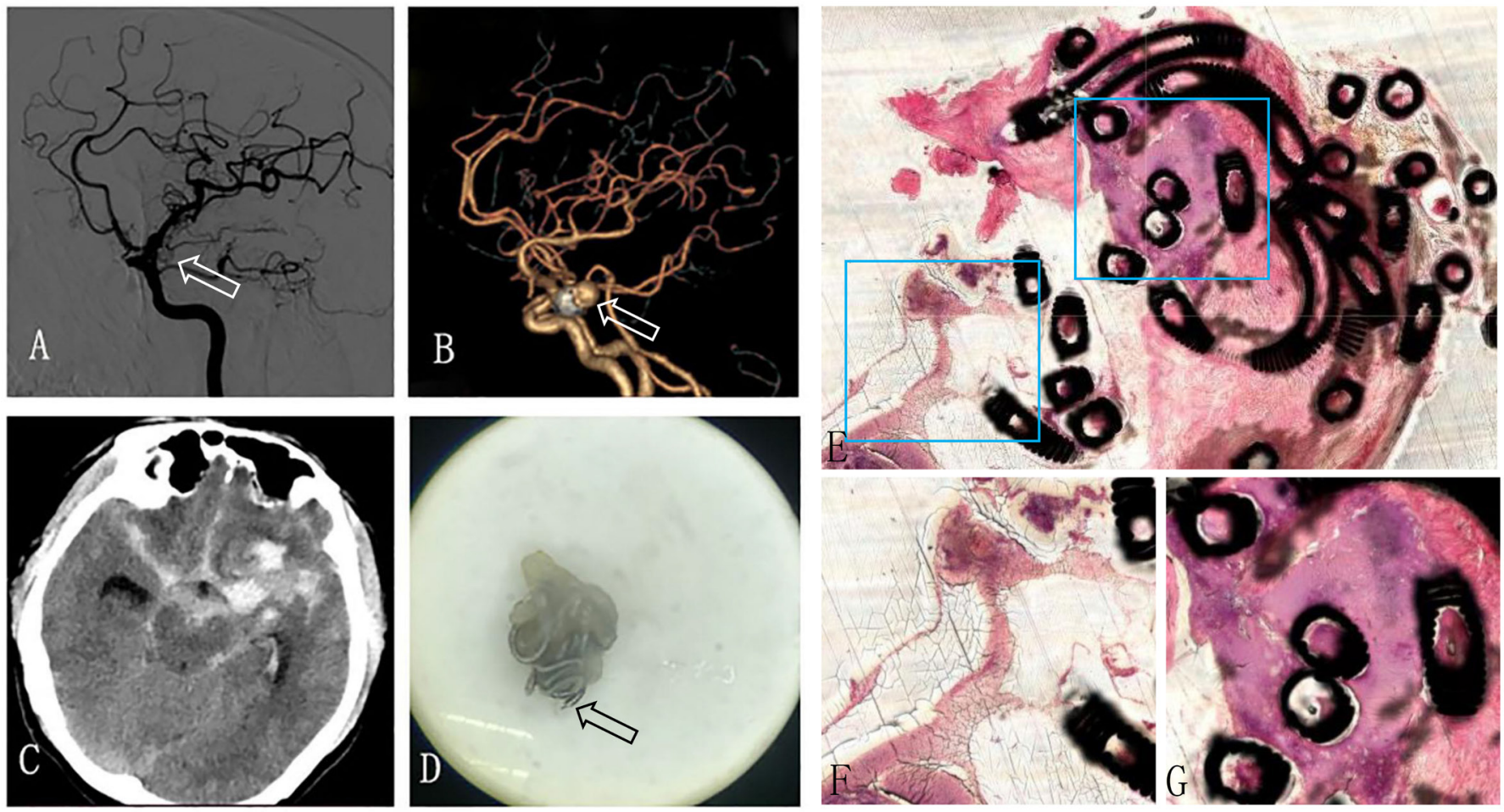

Figure 2 Case 8, patient in their 50s with an aneurysm located at LC7. (A) Digital subtraction angiography reveals complete aneurysm occlusion in LC7 (open arrow). (B) Head CT angiography shows aneurysm recanalization with obvious aneurysm growth (open arrow). (C) Head CT shows subarachnoid hemorrhage from the aneurysm (linear high signal in the basal cistern and longitudinal fissure, patchy heterogeneous high signal shadow in the left frontal base, low signal ring around). (D) Gross specimen with the coils protruding from the aneurysm wall (open arrow). (E-G) Microscopic section (hematoxylin-eosin stain, magnification $\times 5$ and $\times 12.5$ ) showing a fresh thrombus loosely linked with coils (open arrow) and surrounding serum effusion and necrotic collagen fibers (arrow).

that the fresh thrombus gradually turned into scar tissue in the process of thrombus organization. All three types of thrombus found in the present study (fresh thrombus, unstable granulation tissue, and scar tissue) have been described in previous publications. ${ }^{18} 19$ Previous studies of autopsy pathology sections have showed that the fresh thrombus is loosely filled with coils at
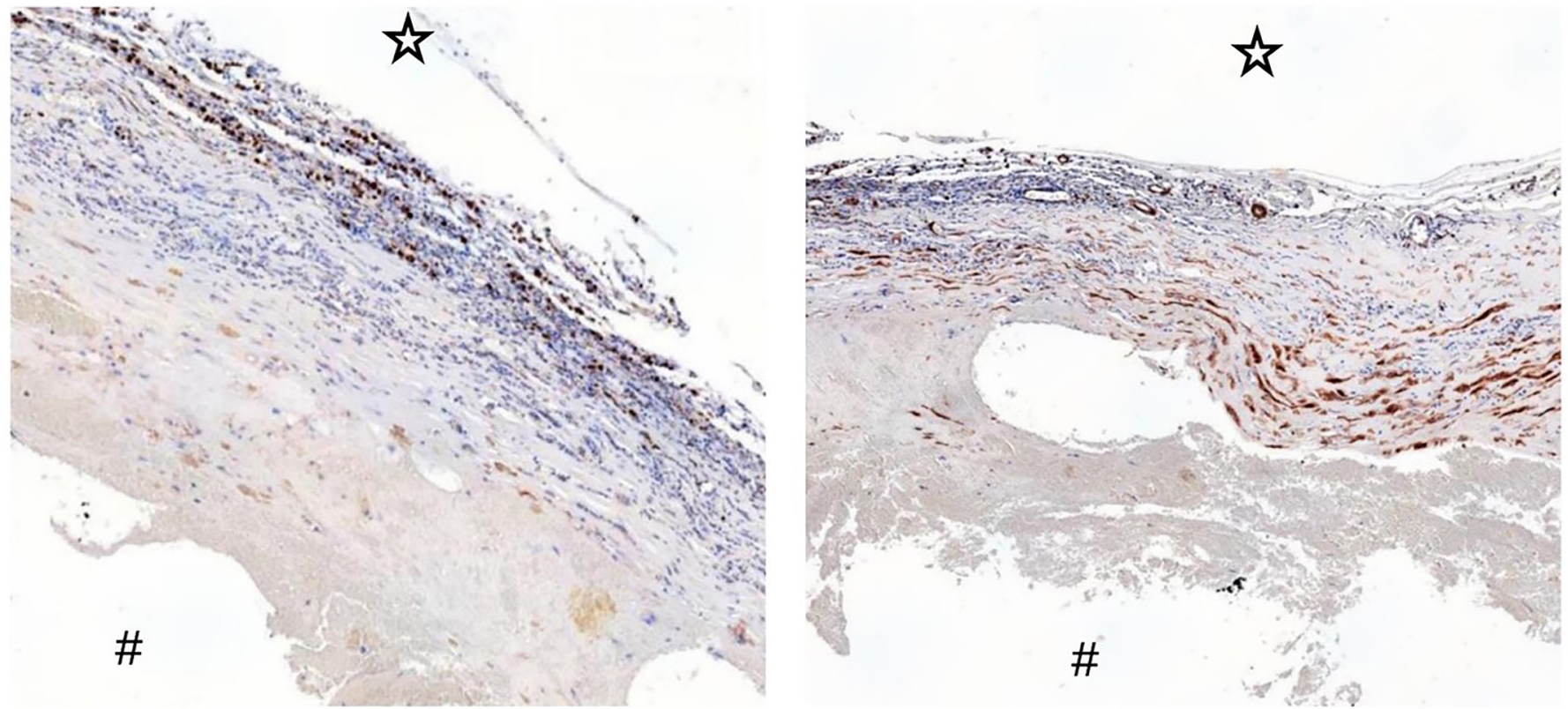

Figure 3 Case 4, patient in their 60s with an aneurysm located in the posterior communicating artery. (A) Immunohistochemical CD68 staining (magnification $\times 2.5$ ) shows macrophages infiltrating the adventitia in the aneurysm wall (brown staining). (B) Immunohistochemical smooth muscle actin staining (magnification $\times 25$ ) showing that the region with more damaged smooth muscle cells has more severe infiltration of inflammatory cells (brown staining) ( $\star$ : outside of the aneurysm vessel ) (\#:aneurysma lumen). 
5 days after coiling treatment. At 2 weeks after coiling, the fibrosis is progressing and the thrombus is being transformed into granulation tissue. At 1 year after coiling, the aneurysm sac is full of mature scar tissue. Our study showed similar components of the different thrombus types. Notably, all of these components coexisted in one recurrent post-treatment aneurysm cavity in our study. Each type of thrombus had its specific location inside the recurrent aneurysm: the fresh thrombus was in the aneurysm dome, unstable granulation tissue was near the neck, and scar tissue was in the middle of the aneurysm sac lining the wall. Such pathologic findings have not been previously reported in the literature.

One important pathological finding was the granulation tissue in the residual cavity at the aneurysm neck. The thrombus was still unorganized without epithelialization. Previous examination of IA autopsy sections has shown that after successful endovascular coil embolization, the vascularized connective tissue completely fills the aneurysm cavity and embeds the coils, and the aneurysm neck is completely covered by a layer of long slender cells, suggesting endothelium. ${ }^{18}$ Our pathological results of recurrent post-embolic aneurysms were different from those of stable postembolic aneurysms. Inadequate neck thrombus fibrosis may be an important indicator of aneurysm recanalization after embolization, and this pathological characteristic in the aneurysmal neck at a certain time after embolization may predict the final treatment outcome. Furthermore, during the operation, we found that this empty cavity was also the site where blood flow continuously impinged into the aneurysm neck. This indicates that the lack of endothelialization may be associated with the empty cavity in the aneurysm neck. A lack of sufficient endothelialization to protect the unorganized thrombus in the aneurysm sac may result in post-embolic aneurysm recurrence. Previous studies have used gadolinium-enhanced 7T MRI to analyze the aneurysm microstructures, including the thrombus and aneurysm wall. ${ }^{20}$ Furthermore, the pathological results of the aneurysm sac thrombus treated by bare platinum coils are correlated with an abnormal signal on 7T MRI in an animal model. ${ }^{21}$ Noninvasive imaging detection of thrombi may help in the diagnosis of recurrent IAs in the future.

Our immunohistochemical staining results provided evidence to suggest that the mechanism of IA recurrence is impairment of the SMCs and infiltration of inflammatory cells. The discontinued and incomplete middle layer in specimens immunohistochemically stained for SMA suggests the impairment of SMCs. Marbacher $e t a^{22}$ found that thrombus organization is significantly dependent on the presence of healthy SMCs in an animal aneurysm model. Similarly, SMC impairment may contribute to the recurrence of human aneurysms, but the detailed and complex mechanisms are unclear. In our study, the integrity and continuity of SMA staining was poorer in regions with more significant macrophage infiltration, suggesting that inflammatory cells may injure the SMCs. This phenomenon was also found in a previous animal study, which reported that the macrophage infiltration resulted in apoptosis of the SMCs in interleukin-1 $\beta$ deficient mice. ${ }^{23}$ Furthermore, both H\&E and CD68 + staining showed the presence of lymphocytes and macrophages in the recurrent aneurysm wall, suggesting persistent chronic inflammation in the wall. The infiltration of inflammatory cells is the basic reaction of coil implantation therapy. Based on the current evidence, the specific role of inflammatory cells in complete aneurysm embolization remains controversial.

In the present study, the mean recurrent aneurysm size was $10.4 \mathrm{~mm}$ and the mean neck width was $3.9 \mathrm{~mm}$. Furthermore, the most common aneurysm recurrence types were self-growth and coil compaction. The basic characteristics of aneurysms affect the outcome of endovascular treatment, with higher rates of recurrence reported for aneurysms with a wide neck and large size. ${ }^{24}$ These imaging characteristics play a role in aneurysm recurrence, and the detailed correction procedures required have been investigated. ${ }^{5725}$

To explain the phenomenon of aneurysm rupture after coiling, we propose two explanations based on (1) the imaging findings of three self-growth aneurysms after coiling, (2) the intraoperative observation of coils projecting at the rupture site, and (3) the histopathological findings. The first explanation is that the aneurysm growth results in thinning of the aneurysm wall and insufficient stress or tension to maintain its own geometric structure. Pathologically, these types of aneurysm have a more unorganized thrombus and less endothelialization to resist blood impingement than other aneurysms, which eventually leads to aneurysm recurrence and rupture. The second explanation is the formation of a pseudocapsule at the rupture point after the first bleeding episode, so that the wall of the pseudocapsule is much thinner and more prone to rupture at the same site. The specific aneurysm re-rupture mechanism after endovascular therapy still needs further research.

\section{LIMITATIONS}

This study had some limitations. Because the specimens were collected from living humans, some aneurysm necks could not be completely obtained. Additionally, in this study, we lacked sufficient cases of unruptured aneurysms so that we were unable to compare the histopathology and morphology of unruptured and ruptured aneurysms. Furthermore, as we did not investigate the corresponding mechanisms, we cannot explain whether inflammation and SMCs directly or indirectly affect the recurrence process.

\section{CONCLUSION}

The nine recurrent aneurysm specimens had particular histopathological features. The coexistence of three types of thrombi is their main characteristic. The formation of stable thrombus may be one of the key points of aneurysm recurrence. Furthermore, SMC damage and infiltration of inflammatory cells in the aneurysm wall probably contribute to the recanalization.

\section{Twitter Ying Zhang @Ying Zhang}

Acknowledgements We thank the patients who participated in this study.

Contributors CW cleaned and analyzed the data; drafted and revised the paper. $X Y, H C, M L$ revised the paper. YZ wrote the statistical analysis plan, cleaned and analyzed the data, and revised the paper. DZ designed data collection tools, monitored data collection for the whole trial, and revised the paper.

Funding This work was supported by National Natural Science Foundation of China (grant numbers: 81671139).

Competing interests There are no conflicts of interest

\section{Patient consent for publication Not required.}

Ethics approval The ethics committee of Beijing Tiantan Hospital approved this study and a statement that participants gave informed consent before taking part. The number of the approval KY2017-017-01.

Provenance and peer review Not commissioned; externally peer reviewed.

Data availability statement Data sharing not applicable as no datasets generated and/or analysed for this study.

Supplemental material This content has been supplied by the author(s). It has not been vetted by BMJ Publishing Group Limited (BMJ) and may not have been peer-reviewed. Any opinions or recommendations discussed are solely those of the author(s) and are not endorsed by BMJ. BMJ disclaims all liability and responsibility arising from any reliance placed on the content. Where the content includes any translated material, BMJ does not warrant the accuracy and reliability 
of the translations (including but not limited to local regulations, clinical guidelines, terminology, drug names and drug dosages), and is not responsible for any error and/or omissions arising from translation and adaptation or otherwise.

Open access This is an open access article distributed in accordance with the Creative Commons Attribution Non Commercial (CC BY-NC 4.0) license, which permits others to distribute, remix, adapt, build upon this work non-commercially, and license their derivative works on different terms, provided the original work is properly cited, appropriate credit is given, any changes made indicated, and the use is non-commercial. See: http://creativecommons.org/licenses/by-nc/4.0/.

ORCID iD

Xinjian Yang http://orcid.org/0000-0002-3076-2933

\section{REFERENCES}

1 Nieuwkamp DJ, Setz LE, Algra A, et al. Changes in case fatality of aneurysmal subarachnoid haemorrhage over time, according to age, sex, and region: a metaanalysis. Lancet Neurol 2009;8:635-42.

2 Etminan N, Rinkel GJ. Unruptured intracranial aneurysms: development, rupture and preventive management. Nat Rev Neurol 2016;12:699-713.

3 Molyneux AJ, Birks J, Clarke A, et al. The durability of endovascular coiling versus neurosurgical clipping of ruptured cerebral aneurysms: 18 year follow-up of the UK cohort of the International Subarachnoid Aneurysm Trial (ISAT). The Lancet 2015:385:691-7.

4 Molyneux AJ, Kerr RSC, Yu L-M, et al. International Subarachnoid Aneurysm Trial (ISAT) of neurosurgical clipping versus endovascular coiling in 2143 patients with ruptured intracranial aneurysms: a randomised comparison of effects on survival, dependency, seizures, rebleeding, subgroups, and aneurysm occlusion. Lancet 2005;366:809-17.

5 Abdihalim M, Watanabe M, Chaudhry SA, et al. Are coil compaction and aneurysmal growth two distinct etiologies leading to recurrence following endovascular treatment of intracranial aneurysm? J Neuroimaging 2014;24:171-5.

6 Cho YD, Lee JY, Seo JH, et al. Does stent implantation improve the result of repeat embolization in recanalized aneurysms? Neurosurgery 2012;71:ons253-9; discussion ons259.

7 Raymond J, Guilbert François, Weill A, et al. Long-term angiographic recurrences after selective endovascular treatment of aneurysms with detachable coils. Stroke 2003;34:1398-403.

8 Raymond J, Darsaut TE. An approach to recurrent aneurysms following endovascular coiling. J Neurointerv Surg 2011;3:314-8.

9 Hasan DM, Nadareyshvili Al, Hoppe AL, et al. Cerebral aneurysm sac growth as the etiology of recurrence after successful coil embolization. Stroke 2012;43:866-8.
10 Cho YD, Jeon JP, Yoo DH, et al. Growth-related major recanalization of coiled aneurysms: incidence and risk factors. Neurosurgery 2018;82:185-91.

11 Boecher-Schwarz HG, Ringel K, Kopacz L, et al. Ex vivo study of the physical effect of coils on pressure and flow dynamics in experimental aneurysms. AJNR Am J Neuroradiol 2000;21:1532-6.

12 Ozawa T, Tamatani S, Koike T, et al. Histological evaluation of endothelial reactions after endovascular coil embolization for intracranial aneurysm. Clinical and experimental studies and review of the literature. Interv Neuroradiol 2003;9:69-82.

13 Frösen J. Smooth muscle cells and the formation, degeneration, and rupture of saccular intracranial aneurysm wall-a review of current pathophysiological knowledge. Trans/ Stroke Res 2014;5:347-56.

14 Xu Z, Rui Y-N, Hagan JP, et al. Intracranial aneurysms: pathology, genetics, and molecular mechanisms. Neuromolecular Med 2019;21:325-43.

15 Yu L-B, Yang X-J, Zhang Q, et al. Management of recurrent intracranial aneurysms after coil embolization: a novel classification scheme based on angiography. J Neurosurg 2018:1-7.

16 Gibson-Corley KN, Olivier AK, Meyerholz DK. Principles for valid histopathologic scoring in research. Vet Pathol 2013;50:1007-15

17 Kakalis NMP, Mitsos AP, Byrne JV, et al. The haemodynamics of endovascular aneurysm treatment: a computational modelling approach for estimating the influence of multiple coil deployment. IEEE Trans Med Imaging 2008;27:814-24.

18 Groden C, Hagel C, Delling G, et al. Histological findings in ruptured aneurysms treated with GDCs: six examples at varying times after treatment. AJNR Am J Neuroradiol 2003:24:579-84.

19 Brinjikji W, Kallmes DF, Kadirvel R. Mechanisms of healing in coiled intracranial aneurysms: a review of the literature. AJNR Am J Neuroradiol 2015;36:1216-22.

20 Zhang C, Chaudhary N, Gemmete JJ, et al. Correlation of thrombus formation on 7 T MRI with histology in a rat carotid artery side wall aneurysm model. J Neurointerv Surg 2014;6:780-4.

21 Sato T, Matsushige T, Chen B, et al. Wall contrast enhancement of thrombosed intracranial aneurysms at 7T MRI. AJNR Am J Neuroradiol 2019;40:1106-11.

22 Marbacher S, Frösén J, Marjamaa J, et al. Intraluminal cell transplantation prevents growth and rupture in a model of rupture-prone saccular aneurysms. Stroke 2014;45:3684-90

23 Moriwaki T, Takagi Y, Sadamasa N, et al. Impaired progression of cerebral aneurysms in Interleukin-1 $\beta$-deficient mice. Stroke 2006;37:900-5.

24 Henkes H, Fischer S, Weber W, et al. Endovascular coil occlusion of 1811 intracranial aneurysms: early angiographic and clinical results. Neurosurgery 2004;54:268-85.

25 Chalouhi N, Bovenzi CD, Thakkar V, et al. Long-term catheter angiography after aneurysm coil therapy: results of 209 patients and predictors of delayed recurrence and retreatment. J Neurosurg 2014;121:1102-6. 